\title{
All-optical sampling and spectrographic pulse measurement using cross-absorption modulation in multiple-quantum-well devices
}

\author{
Douglas Reid, ${ }^{1, *}$ Paul J. Maguire, ${ }^{1}$ Liam P. Barry, ${ }^{1}$ Quang-Trung Le, ${ }^{2}$ Sebastian Lobo, ${ }^{2}$ Mathilde Gay, ${ }^{2}$ Laurent Bramerie, ${ }^{2}$ \\ M. Joindot, ${ }^{2}$ Jean-Claude Simon, ${ }^{2}$ D. Massoubre, ${ }^{3}$ Jean-Louis Oudar, ${ }^{3}$ and Guy Aubin ${ }^{3}$ \\ ${ }^{1}$ Research Institute for Networks and Communications Engineering, Dublin City University, Dublin 9, Ireland \\ ${ }^{2}$ FOTON-ENSSAT/PERSYST Platform, 6 Rue de Kerampont, BP 80518 22305, Lannion Cedex, France \\ ${ }^{3}$ LPN-CNRS, Route de Nozay, F-91460, Marcoussis, France \\ *Corresponding autor: douglas.reid@eeng.dcu.ie
}

Received November 2, 2007; revised March 6, 2008; accepted April 1, 2008;

posted April 18, 2008 (Doc. ID 89244); published May 23, 2008

\begin{abstract}
The application of cross absorption modulation for optical sampling and phase sensitive pulse measurement is presented. Both a commercial $40 \mathrm{GHz}$ electroabsorption modulator and a vertical microcavity saturable absorber are used to sample a $2 \mathrm{ps}$ pulse train at $40 \mathrm{GHz}$. The modulator was then used to make crossabsorption modulation based frequency-resolved optical gating (FROG) measurements. The results were verified by comparison with a commercial optical sampling oscilloscope and the more standard second harmonic generation FROG technique. (C) 2008 Optical Society of America OCIS codes: $060.2330,320.7100$.
\end{abstract}

\section{INTRODUCTION}

With increasing line rates the measurement of optical intensities on very short time scales is very important. The traditional approach of optical to electrical conversion using a photodiode and electrical sampling is limited to the bandwidth of the detector, which is typically $50 \mathrm{GHz}$. One solution to this is to employ optical sampling techniques. All-optical sampling offers the potential for measurements with much higher resolution, being limited only by the duration and jitter of the sampling pulse and the speed of the nonlinear effect used to implement them.

In addition to measurement of the time varying intensity, for many applications, measurement of the phase is also desirable. This is of increasing importance in optical communication with the recent interest in advanced modulation formats, which utilize phase modulation [1,2]. It also has application in the study of nonlinear optics, laser physics, dispersion management, and all-optical processing technologies. Many techniques have been developed to carry out these measurements. In particular spectrographic techniques such as frequency-resolved optical gating (FROG) [3] and interferometric techniques such as spectral phase interferometry for direct electricfield reconstruction (SPIDER) [4] have seen wide spread application.

The absorption saturation effect due to band filling in semiconductors has been used to great advantage in producing ultrashort pulse mode locked lasers [5] and for the regeneration of optical communications channels [6]. This effect can also be exploited for all-optical sampling of high-repetition-rate optical channels since a short pump pulse at one wavelength can be used to generate a short temporal gate for a signal at another wavelength. This is referred to as cross-absorption modulation (XAM). XAM has been used for the switching of entire pulses for the purposes of optical wavelength conversion $[7,8]$ and also for optical regeneration $[9,10]$ but has seen little or no application in the measurement of intensity waveforms.

Here two novel setups for optical sampling based on $\mathrm{XAM}$ in multiple-quantum-well (MQW) semiconductor devices are presented; both have the advantage of being polarization insensitive, contain no moving parts or freespace optics, and offer the potential for integration. This is further extended with the concept and implementation of a new FROG technique based on XAM in an electroabsorption modulator (EAM). The technique is simple, polarization insensitive, and requires minimal pulse energy.

This paper is divided as follows: Section 2 introduces the principles of all-optical sampling and gives an overview of some of the sampling techniques, which have already been reported. Section 3 will describe how optical sampling can be carried out using XAM in a vertical microcavity saturable absorber. The results are compared with measurements made using a commercial optical sampling oscilloscope [(OSO) from Picosolve Inc.]. Section 4 presents how similar measurements can be made with a commercial EAM. In Section 5 the work is extended to complete phase sensitive pulse measurement with the inclusion of an OSA following the XAM sampling setup.

\section{OPTICAL SAMPLING}

To evaluate the performance of the next generation of high-speed optical communications systems, future pulse characterization techniques will require a subpicosecond resolution and a low (subgigahertz) sampling rate. This avoids the need for high-speed electronics and allows individual channel data rates in excess of $500 \mathrm{Gbit} / \mathrm{s}$ to be 
monitored. This performance can be achieved using an optical sampling system. The principle of optical sampling is shown in Fig. 1. The sampling and signal pulses are optically combined using a passive fiber coupler, before being incident on a nonlinear optical material (acting as a nonlinear sampling gate). The nonlinear medium allows the selection or gating of the small section of the signal under test, which coincides with the sampling pulse. A bandpass filter is also sometimes required to remove the sampling pulse so that only the gated signal falls on the detector. The delay between the sampling pulse and the signal under test is varied either electrically or optically, thereby allowing different sections of the signal to be evaluated. A large variety of nonlinear optical processes have been investigated for this application including self-phase modulation (SPM) in nonlinear amplifying loop mirrors (NALMs) [11], four-wave mixing (FWM) both in fiber [12] and semiconductor optical amplifiers (SOAs) [13], sum frequency generation (SFG) in $\mathrm{LiNbO}_{3}$ [14], and two photon absorption (TPA) [15,16]. These techniques have proven highly successful but do suffer from some drawbacks. They are generally polarization sensitive or require complicated techniques to allow for polarization diversity. They also have high power requirements, generally needing hundreds of milliwatts of peak power and often have delicate free-space optics or long lengths of optical fiber. This paper focuses on the use of XAM as a nonlinear process for optical sampling. This process has the advantages of being polarization insensitive and having very low power/interaction length requirements.

\section{SAMPLING USING A MULTIPLE- QUANTUM-WELL VERTICAL MICROCAVITY SATURABLE ABSORBER}

Two different nonlinear elements were used in the sampling experiments. The first was a nonlinear mirror based on a vertical microcavity saturable absorber. The saturable absorber was packaged in a module with eight input channels, and only one channel is used in our experiment [6]. The saturable absorber structure is shown in Fig. 2(a); it contains seven metal-organic chemical-vapor deposition (MOCVD)-grown InGaAs/InP quantum wells embedded in a microresonator. The resonator reduces the device saturation threshold and increases the saturation contrast. The quantum wells are located at the antinodes of the intracavity intensity. The bottom mirror is a broadband high-reflectivity metallic-based mirror (Ag) and the top mirror is a multilayer dielectric mirror $\left(2 \times\left[\mathrm{TiO}_{2} / \mathrm{SiO}_{2}\right]\right)$. The time scale for the absorption saturation in the quantum wells is related to the carrier recombination time, which in this case was shortened to

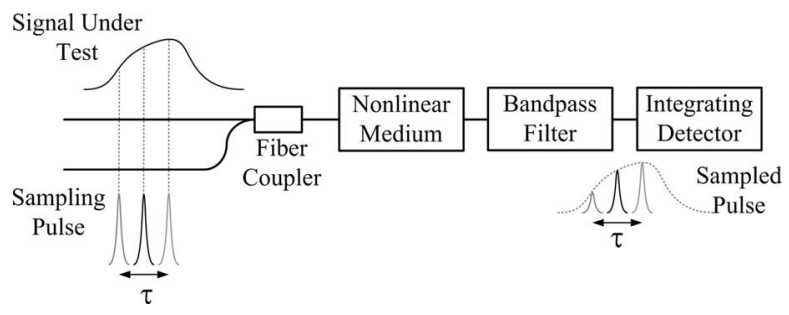

Fig. 1. Principle of optical sampling.

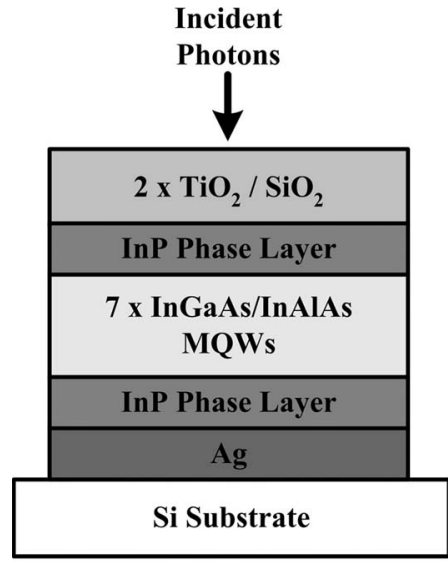

(a)

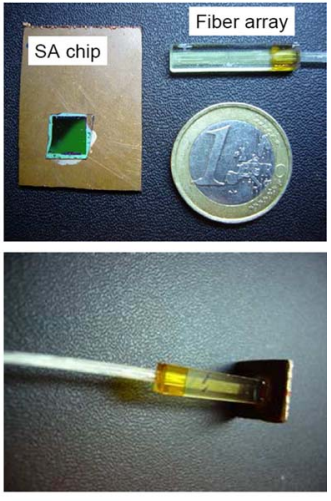

(b)
Fig. 2. (Color online) (a) Saturable absorber vertical microcavity structure. (b) Saturable absorber chip and eight-fiber array used in coupling.

$5 \mathrm{ps}$ by the introduction of recombination sites through heavy-ion-irradiation. The device operates in a reflective mode, the reflectivity being small at low signal levels and high at high signal levels. A fiber array was used to couple the saturable absorber chip to eight standard single-mode fibers (SMF-28) with $250 \mu \mathrm{m}$ spacing. The fiber array was fixed to the mirror with an adhesive such that all eight output beams had a calculated mode field diameter (MFD) of $4.5 \mu \mathrm{m}$ on the surface of the mirror [Fig. 2(b)]. Such a layout would enable a single device to be used for regeneration in a multiwavelength system. The use of the seven additional channels on the saturable absorber would also allow for the possibility of simultaneous and independent sampling of multiple wavelength channels. The simple fabrication process along with the fact that high-speed rf packaging is not required, significantly reduces the cost of the device as a sampling element.

The wavelength-dependent reflectivity of the eight channels is shown in Fig. 3. The dip in the reflectivity at $1547 \mathrm{~nm}$ corresponds to the resonant wavelength of the microcavity. There was only a $1 \mathrm{~nm}$ variation in the position of this dip and less than a $2 \mathrm{~dB}$ variation in the depth of its response, which is evidence of the uniformity of the

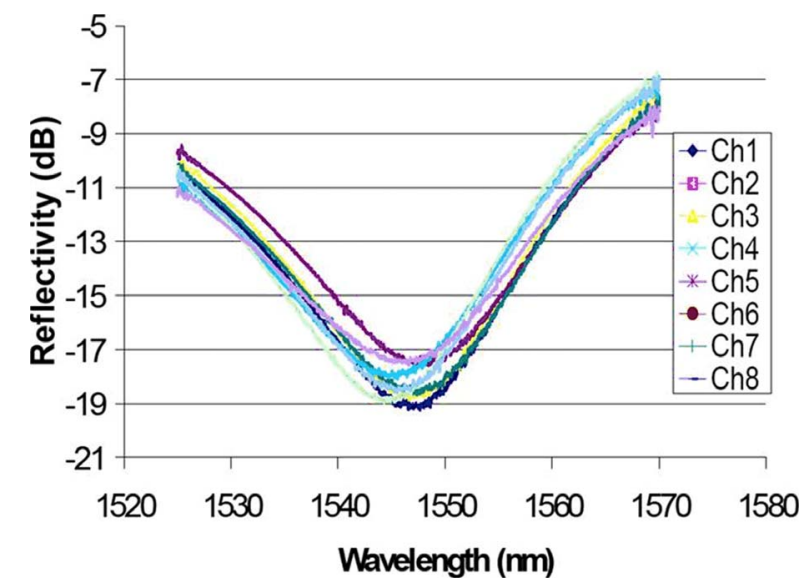

Fig. 3. (Color online) Reflectivity of eight fibers coupled to a saturable absorber, showing resonance dip at $1547 \mathrm{~nm}$ and less than $2 \mathrm{~dB}$ variation between channels. 
coupling and angle of the fibers in the array. The effects of $\mathrm{XAM}$ in the multiple-quantum-well saturable absorber (MQW-SA) are shown in Fig. 4. Two spectra of a cw signal after reflection off the SA are shown. In both cases the cw signal was mixed with a $10 \mathrm{GHz} 2$ ps tunable modelocked laser (TMLL) at $1558 \mathrm{~nm}$, which acts as a sampling pulse. At low sampling pulse powers $(0 \mathrm{dBm})$ there is no modulation of the cw, but increasing the power $(15 \mathrm{dBm})$ results in the broadening and $10 \mathrm{GHz}$ modulation of the $\mathrm{cw}$ signal. The intensity dependent loss of the MQW-SA is shown in Fig. 5. This was measured using the same TMLL at $1547 \mathrm{~nm}$ in conjunction with an erbium-doped fiber amplifier (EDFA). In saturation, the insertion loss of the device is $\sim 7 \mathrm{~dB}$ with $5 \mathrm{~dB}$ of additional loss due to coupling. The unsaturated loss was $19 \mathrm{~dB}$, giving a contrast of $7 \mathrm{~dB}$. In the sampling experiments the signal under test on its own did not have sufficient intensity to saturate the MQW-SA and therefore experiences a large loss when incident on the device $(19 \mathrm{~dB})$. However, in the presence of the sampling pulse the MQW-SA saturates, allowing the signal to be reflected with reduced loss $(12 \mathrm{~dB})$. This allows for the sampling of a short temporal section of the signal under test. Note that the $3 \mathrm{~dB}$ saturation threshold of the MQW-SA is as low as $5 \mathrm{dBm}$ at the operating wavelength and repetition rate; consequently, the sampling can be operated at a high repetition rate (10 GHz in this experiment), opening the possible use of this technique in real-time sampling [17]. The sampling setup used is shown in Fig. 6. The setup employed a $10 \mathrm{GHz} 2 \mathrm{ps}$ sampling pulse from a TMLL at $1558 \mathrm{~nm}$, which was combined (using a 50/50 coupler) with the signal under test. The combined signal was then reflected off the saturable absorber via an optical circulator. An optical filter with a $5 \mathrm{~nm}$ bandwidth centered at $1547 \mathrm{~nm}$ was then used to remove the sampling pulse before the final measurement with a low-speed detector. An optical delay line (ODL) was used to adjust the delay between the sampling pulse and the signal under test, and an EDFA was used to provide the necessary average power in the sampling pulse $(12.7 \mathrm{dBm})$. Computer control of the ODL and detector allowed measurements with a refresh rate of $\sim 1 \mathrm{~Hz}$. The signal under test was generated using a second $10 \mathrm{GHz} 2$ ps TMLL, which was tuned to near the saturable absorbers resonant wavelength $(1547 \mathrm{~nm})$. A

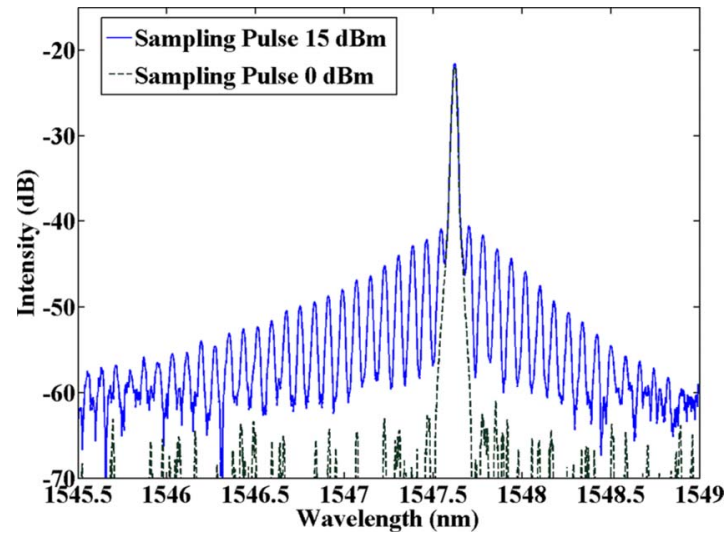

Fig. 4. (Color online) Spectra of a cw signal when copropagated with a $10 \mathrm{GHz} 2 \mathrm{ps}$ sampling pulse and reflected off the MQW-SA.

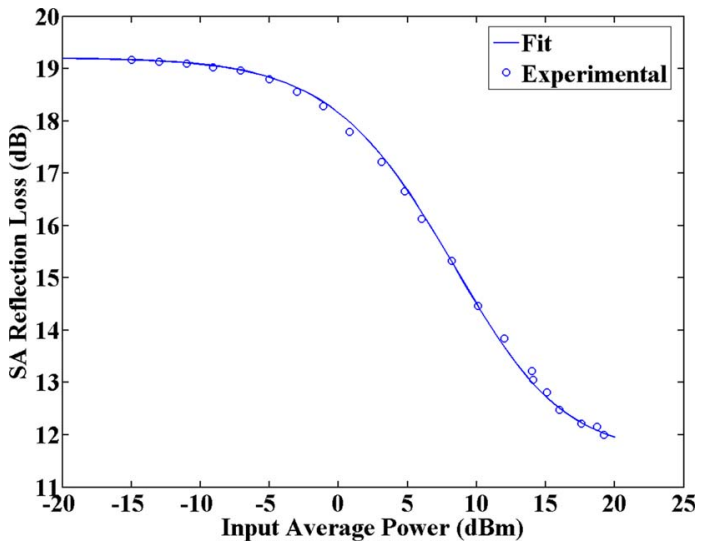

Fig. 5. (Color online) Response of saturable absorber's loss to increasing average input power from a $10 \mathrm{GHz} \sim 2$ ps TMLL pulse at $1547 \mathrm{~nm}$.

$1 \times 4$ optical multiplexer was used to increase the line rate to $40 \mathrm{GHz}$ and allowed the relative position and amplitude of the multiplexed pulses to be adjusted. Variable optical attenuators (VOA) were used on both the sampling and the signal pulses to control the optical power incident on the saturable absorber.

Examples of the measurements of the $40 \mathrm{GHz}$ pulse train using both the MQW-SA and a commercial OSO are given in Fig. 7. In Fig. 7(b) bits 3 and 4 have been attenuated to study the behavior of the optical sampling toward peak-power variations thus highlighting the linearity of the measurement. For the MQW-SA setup the pulse width is overestimated because of the limited response time of the saturable absorber (5 ps) and the short duration of the pulses $(\sim 2 \mathrm{ps})$. This could be improved with the use of a faster saturable absorber such as those reported by Gicquel-Guezo et al. [18], which have response times of $290 \mathrm{fs}$. The XAM measurements have a background due to the finite contrast of the absorption saturation, which is a disadvantage but can be overcome with an initial background measurement. However, this experiment clearly shows the suitability of the technique for optical sampling as the measured ratio in the pulse amplitudes of both techniques are in good agreement. In Fig. 7 the average sampling power was $12.8 \mathrm{dBm}$ while the average signal power was $-10 \mathrm{dBm}$, although measurements were made with reasonable signal to noise ratios with signal powers down to $-20 \mathrm{dBm}$.

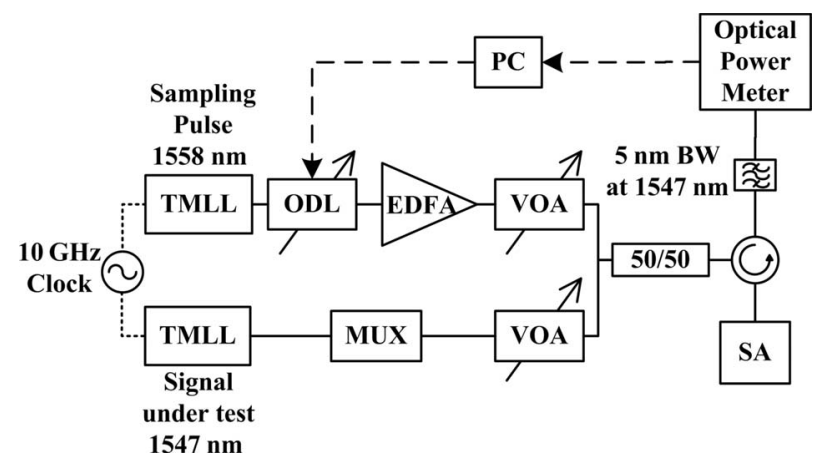

Fig. 6. Experimental setup used for the sampling of a $40 \mathrm{GHz}$ pulse train using a saturable absorber. 


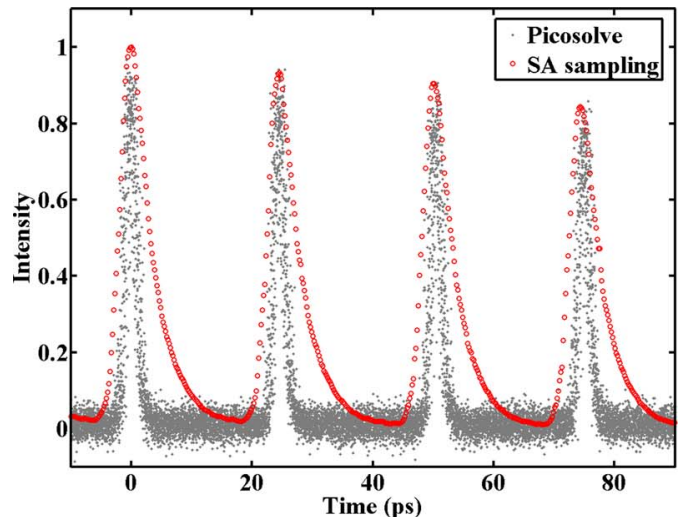

(a)

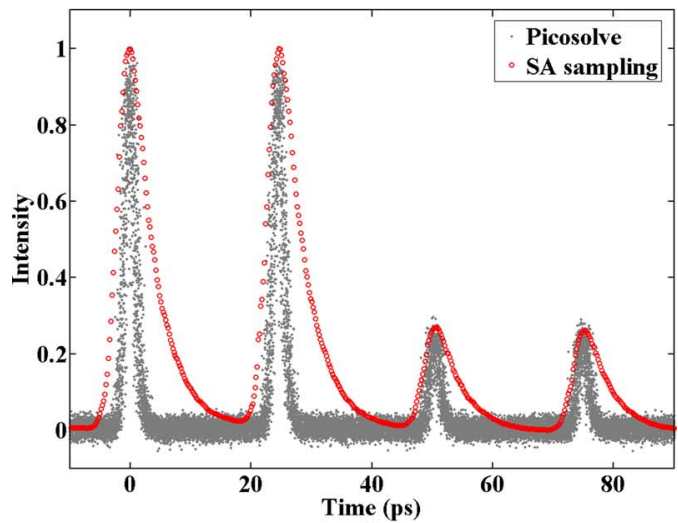

(b)

Fig. 7. (Color online) Sampling of a $40 \mathrm{GHz}$ pulse train using a saturable absorber. Results are compared with a commercial sampling oscilloscope that employs FWM (Picosolve).

\section{SAMPLING USING AN ELECTROABSORPTION MODULATOR}

The second nonlinear element used was a commercial EAM (from OKI). EAMs typically have an unbiased bandgap, which is greater than the energy of the incident photons; with the application of a small negative bias the quantum-confined Stark effect (QCSE) and the FranzKeldysh effect result in the lowering of the effective bandgap and an increase in the absorption. This is commonly exploited to electrically modulate an optical carrier at very high data rates ( $>40 \mathrm{Gbit} / \mathrm{s})$. However if an EAM is negatively biased and exposed to a high-intensity optical pump signal, the electrically induced absorption can be saturated thereby allowing the propagation of the light with reduced absorption. This absorption saturation is due to band filling in the quantum wells and the reduction of the externally applied electric field inside the active region due to photogenerated carriers [8]. As shown in Section 3 this saturation can be used to produce XAM and implement all-optical sampling.

Unlike the MQW-SA described in Section 3 the EAM was operated in transmission rather than reflection. The EAM's intensity dependent transmission is shown in Fig. 8. These measurements were taken using a TMLL with $10 \mathrm{GHz} 2 \mathrm{ps}$ pulses at $1550 \mathrm{~nm}$. The EAM's dc bias was set to $-3 \mathrm{~V}$. The measurements show that the smallsignal loss, for inputs below $-5 \mathrm{dBm}$, was $25 \mathrm{~dB}$. The $3 \mathrm{~dB}$ saturation threshold was at $4 \mathrm{dBm}$, corresponding to a

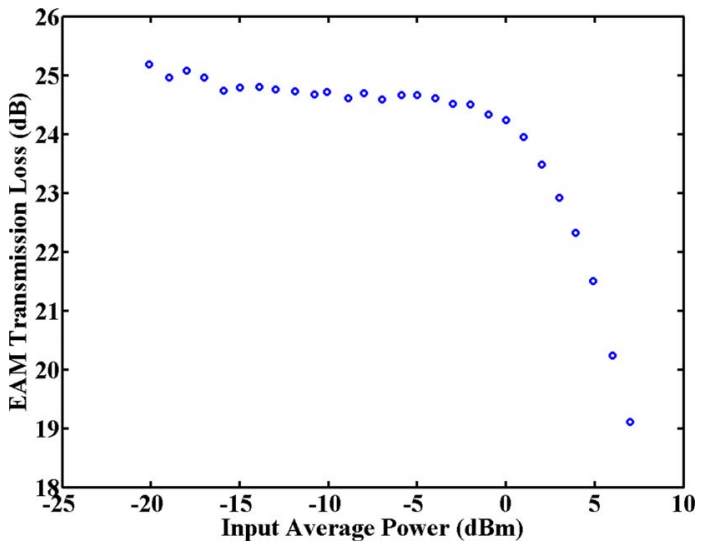

Fig. 8. (Color online) EAM transmission loss as a function of average optical input power from a $10 \mathrm{GHz} \sim 2 \mathrm{ps}$ TMLL pulse at $1550 \mathrm{~nm}$. The EAM was biased at $-3 \mathrm{~V}$.

peak power of $125 \mathrm{~mW}$. The loss had dropped to $19 \mathrm{~dB}$ with an input power of $7 \mathrm{dBm}$, giving a contrast of $6 \mathrm{~dB}$. At this point the EAM's loss had still not completely saturated; however measurements at higher optical powers were not taken due to the risk of damage to the device.

The experimental setup used for sampling is shown in Fig. 9. A TMLL was used to generate a $10 \mathrm{GHz} 2 \mathrm{ps}$ sampling pulse, which was counterpropagated in the EAM with the signal under test. An EDFA was used to give the sampling pulses an average power of $5.3 \mathrm{dBm}$. A $5 \mathrm{~nm}$ bandpass filter centered at $1544 \mathrm{~nm}$ was used before the power meter to remove any residual sampling pulse that passes through the circulator. A computer controlled ODL and optical power meter were used to control the delay between the sampling pulse and the signal under test and take measurements.

A comparison between sampling with the EAM, MQW-SA, and OSO is shown in Fig. 10. The result shows good agreement with the result taken with the MQW-SA but had a slightly longer recovery time of $\sim 7.5 \mathrm{ps}$. Despite the slower speed shown here, EAMs have several advantages over the MQW-SA, including a lower saturation threshold most likely due to better optical confinement. They also have greater potential for integration with other devices such as SOAs in comparison with the vertical cavity structure of the MQW-SA.

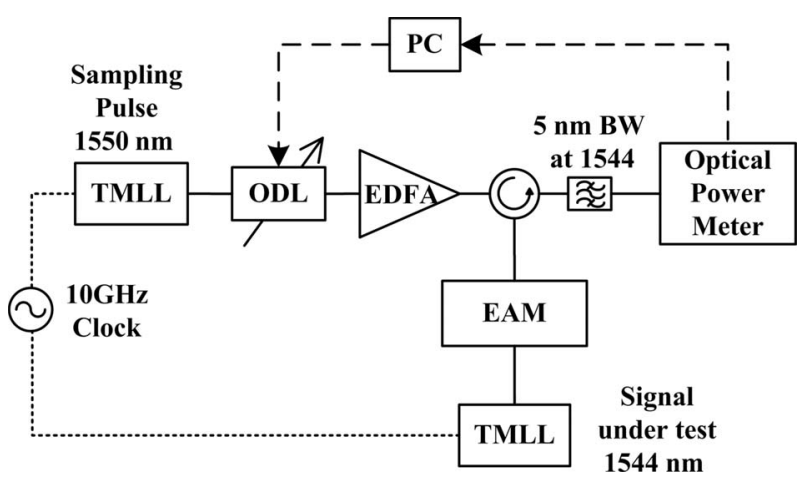

Fig. 9. Experimental setup used for the sampling of a $10 \mathrm{GHz}$ pulse train using an electroabsorption modulator. 


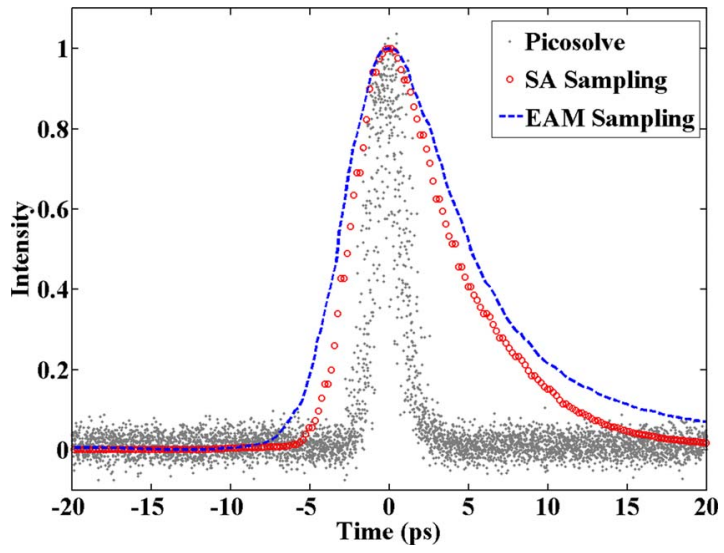

Fig. 10. (Color online) Impulse response of EAM sampling compared with SA sampling and a commercial OSO.

\section{CROSS-ABSORPTION MODULATION FROG}

In this section the sampling setup presented in Section 4 is extended to a full spectrographic measurement. The FROG technique was first presented by Trebino and Kane [3]. The FROG works by measuring a spectrogram of the pulse under test. From this spectrogram it is then possible to extract the complex amplitude (intensity and phase) of the optical pulse, without any assumption about its shape or structure. There are a wide variety of techniques for the measurement of spectrograms, which are distinguished by the gating process they exploit. Many FROG setups use nonlinear crystals and processes such as second harmonic generation (SHG), third harmonic generation (THG), self-diffraction (SD), or polarization gating (PG) to implement the gating [19]. These techniques suffer from being polarization sensitive and require complex free-space alignment. The use of FWM and XPM in both fiber and SOAs has also been demonstrated $[20,21]$; while these techniques overcome the need for bulk optics they are still generally polarization sensitive. More recently the use of high-speed optical modulators to gate the optical pulse before spectral measurement has been shown by Dorrer and Kang [22]. In such systems an electrical signal is used to drive the modulator and generate a gate for the optical signal under test. This has the advantage of much greater sensitivity since no nonlinear optical process is required.

Here XAM in the EAM is used in contrast to the electrical drive used by Dorrer and Kang. This can potentially have the advantage of reducing the requirements on highspeed electrical components and packaging and also enables great versatility in terms of pulse repetition rate. The drawback of this technique is the requirement of an additional optical pulse source, which is synchronized with the signal under test. This could be overcome with a counterpropagating self-gating approach, where the pulse under test is also used as the sampling pulse. However, this would be at the expense of sensitivity.

The XAM-FROG setup used is shown in Fig. 11. This is essentially the same setup as shown in Fig. 9 except the optical power meter has been replaced with an optical spectrum analyzer (OSA). An rf phase shifter was used to control the delay between the two pulse sources, and a

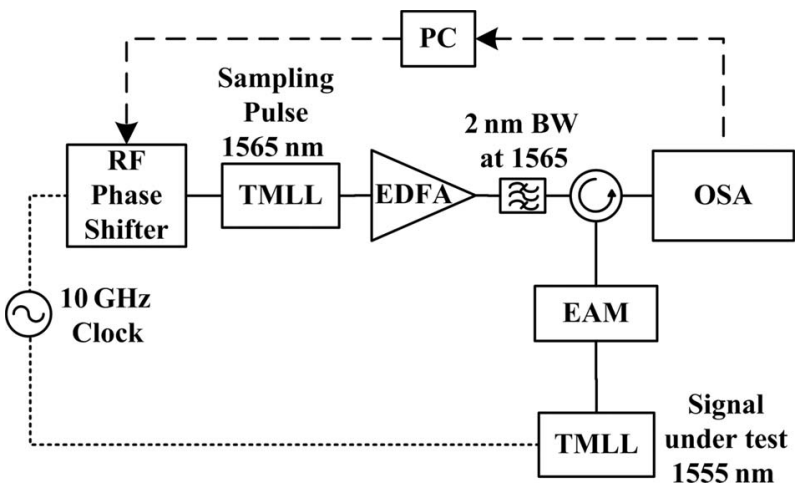

Fig. 11. XAM-FROG setup using an EAM.

$2 \mathrm{~nm}$ filter was added to the sampling arm to stop the small amount of amplified spontaneous emission (ASE) that leaks through the circulator. The bandpass filter previously used after the circulator is no longer required because of the wavelength selectivity of the OSA. The sampling pulse, at $1565 \mathrm{~nm}$, had an average power of $+5 \mathrm{dBm}$ while the signal under test, at $1555 \mathrm{~nm}$, had an average power of $-5 \mathrm{dBm}$. Measurements could be made with significantly less signal power if the full sensitivity of the OSA was exploited, but this would have increased the time for each measurement (currently approximately $2 \mathrm{~min}$ ). The spectrograms were $256 \times 256$ points and were measured by successively stepping the delay between the sampling pulse and the signal under test and taking a spectrum at each delay. An example of the measured spectrograms is shown in Fig. 12. The spectrograms were inverted to extract the complex amplitudes of the signal under test and the gate function using a principal component generalized projection algorithm implemented using the power method [23]. The spectrum of the gate function (measured previously) was used initially as an additional constraint in the recovery.

The TMLL pulse measured using the XAM-FROG is shown in Fig. 13. To verify this result the same pulse was also measured using the more common SHG-FROG technique, using a setup consisting of a noncolinear Michelson interferometer in conjunction with a lithium niobate crystal with a thickness of $1 \mathrm{~mm}$ to form the autocorrelator. This was followed by a $300 \mathrm{~mm}$ double-pass CzernyTurner spectrometer with a 1200 lines/mm diffraction

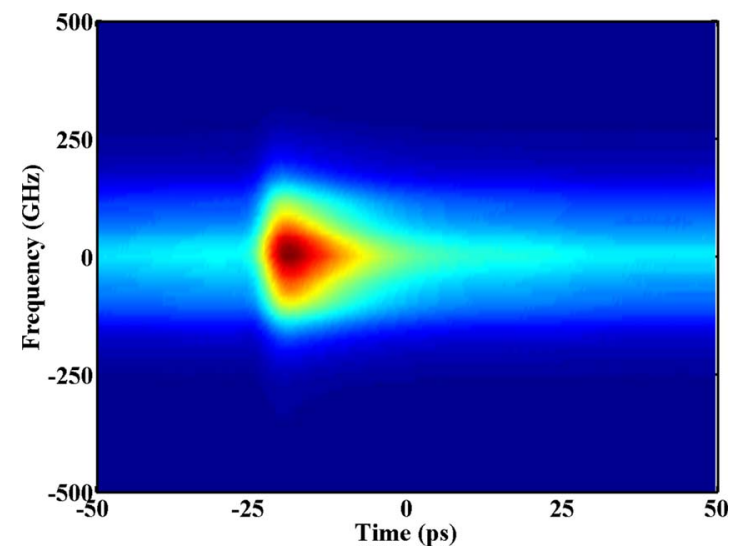

Fig. 12. (Color online) Spectrogram measured using XAM-FROG. 


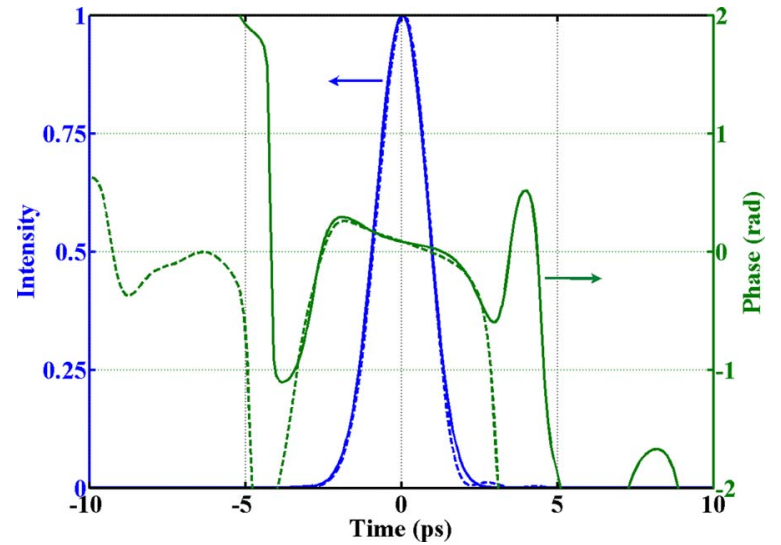

Fig. 13. (Color online) TMLL intensity (left) and phase (right) profile measured by XAM-FROG (solid) and SHG-FROG (dashed).

gating and a 1024 element CCD array. The resulting SHG-FROG apparatus had a delay resolution of $16 \mathrm{fs}$ $(1 \mu \mathrm{m})$ and a spectral resolution of $0.025 \mathrm{~nm}$ at $775 \mathrm{~nm}$. The SHG-FROG required $10 \mathrm{dBm}$ of average power to perform the same measurement $(15 \mathrm{~dB}$ more than the XAM-FROG). The spectrogram recovery errors were $1.8 \times 10^{-3}$ and $2.4 \times 10^{-3}$ for the SHG and XAM FROGs, respectively, indicating reasonable recovery accuracy. The results show good agreement with the SHG-FROG only giving a slightly shorter pulse of $1.92 \mathrm{ps}$ compared with 1.95 from the XAM-FROG. The time bandwidth products measured were 0.48 and 0.45 for the SHG and XAM FROG, respectively. This discrepancy could be because of the slightly more limited spectral resolution in the SHG-FROG setup $(\sim 12.5 \mathrm{GHz})$ as compared to the resolution of the OSA used in the XAM-FROG $(8.75 \mathrm{GHz})$. As further confirmation of the measurement accuracy, the recovered pulse spectrum was compared with an OSA measurement. This comparison is shown in Fig. 14 and again shows very good agreement. In addition, for recovery of the pulse under test, amplitude and phase of the EAMs response was also measured. The gate supplied by the EAM is shown in Fig. 15. The loss is seen to saturate by $4.5 \mathrm{~dB}$ and recovers in $13.5 \mathrm{ps}$. There is also a corresponding phase shift due to the changes in the carrier density in the quantum wells. Although the gate supplied by the XAM does not have high extinction, resulting in a spec-

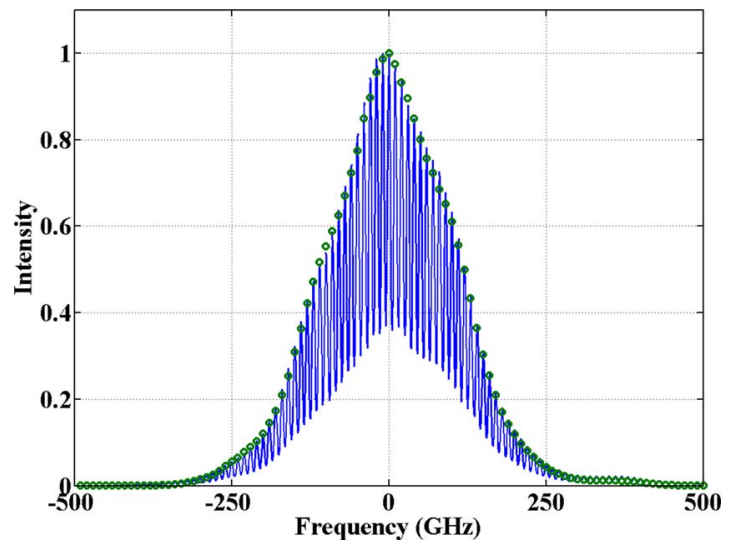

Fig. 14. (Color online) Recovered pulse spectrum (circles) compared with an OSA measurement (solid).

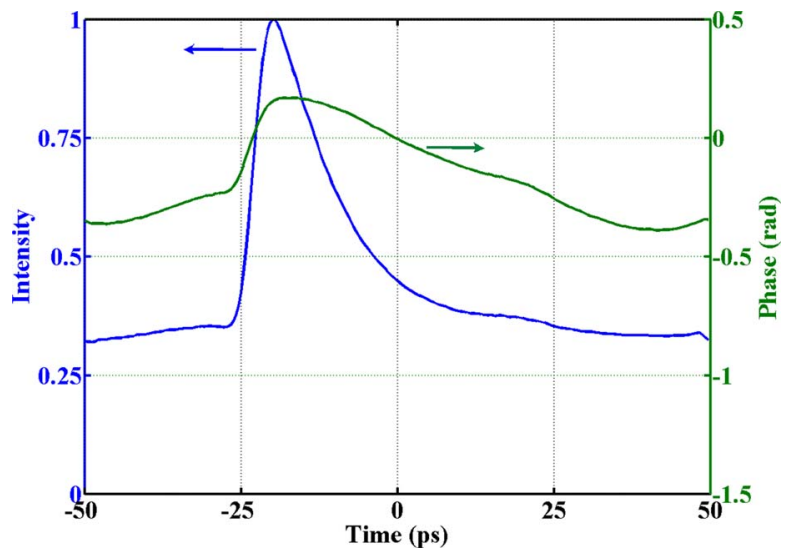

Fig. 15. (Color online) XAM gate function supplied by optically pumped EAM.

trogram, which does not go to zero at its edges, it has been shown that this is not necessarily required to make an accurate measurement, and in fact a pure phase modulation can be used as a spectrographic gate [24].

The XAM-FROG has several advantages over conventional nonlinear FROG techniques including its simplicity, polarization insensitivity, lack of free-space optics, and low peak-power requirements. It is also an alternative to the linear spectrographic setup presented by Dorrer and Kang, having comparible sensitivity without the requirement of an optical modulator with high-speed packaging or rf drive circuitry. The selection of which technique is best suited to a given application would depend on the availability of optical pulse sources or high-speed electrical clocks and electronics.

\section{CONCLUSION}

We present the application of XAM in MQW semiconductor devices for all-optical sampling and spectrographic measurement of short high-repetition-rate pulse sources. Two simple optical sampling setups-one employing a vertical microcavity saturable absorber, the other using a commercial EAM-were demonstrated and used to measure a $40 \mathrm{GHz}$ pulse train. The setups had several advantages over other techniques including their simplicity, sensitivity, polarization insensitivity, and the potential for integration. The results were compared with a commercial sampling oscilloscope and showed good agreement. The use of the seven additional channels on the saturable absorber also allows for the possibility of simultaneous and independent sampling of multiple wavelength channels. By employing a faster saturable absorber this technique offers the potential to implement a simple optical sampling oscilloscope with a bandwidth approaching $500 \mathrm{GHz}$. For the first time a FROG technique, which exploits XAM, was also implemented and used to measure a $1.9 \mathrm{ps} 10 \mathrm{GHz}$ pulse. The result was verified with a conventional SHG-FROG and showed good agreement.

\section{REFERENCES}

1. T. Kawanishi, T. Sakamoto, T. Miyazaki, M. Izutsu, T. Fujita, S. Mori, K. Higuma, and J. Ichikawa, "High-speed optical DQPSK and FSK modulation using integrated 
Mach-Zehnder interferometers," Opt. Express 14, 4469-4478 (2006).

2. C. Jin-Xing, C. R. Davidson, M. Nissov, L. Haifeng, W. T. Anderson, C. Yi, L. Li, A. N. Pilipetskii, D. G. Foursa, W. W. Patterson, P. C. Corbett, A. J. Lucero, and N. S. Bergano, "Transmission of $40-\mathrm{Gb} / \mathrm{s}$ WDM signals over transoceanic distance using conventional NZ-DSF with receiver dispersion slope compensation," J. Lightwave Technol. 24, 191-200 (2006).

3. D. J. Kane and R. Trebino, "Characterization of arbitrary femtosecond pulses using frequency-resolved optical gating," IEEE J. Quantum Electron. 29, 571-579 (1993).

4. C. Iaconis and I. A. Walmsley, "Spectral phase interferometry for direct electric-field reconstruction of ultrashort optical pulses," Opt. Lett. 23, 792-794 (1998).

5. U. Keller, K. J. Weingarten, F. X. Kartner, D. Kopf, B. Braun, I. D. Jung, R. Fluck, C. Honninger, N. Matuschek, and J. Aus der Au, "Semiconductor saturable absorber mirrors (SESAM's) for femtosecond to nanosecond pulse generation in solid-state lasers," IEEE J. Sel. Top. Quantum Electron. 2, 435-453 (1996).

6. L. Bramerie, Q. T. Le, S. Lobo, M. Gay, M. Joindot, J. C. Simon, A. Poudoulec, M. Van der Keur, C. Devemy, D. Massoubre, J.-L. Oudar, G. Aubin, A. Shen, and J. Decobert, "Cascadability and wavelength tunability assessment of a $2 \mathrm{R}$ regeneration device based on a 8 channel saturable absorber module," in National Fiber Optic Engineers Conference, OSA Technical Digest Series (CD) (Optical Society of America, 2007), paper PDP1.

7. S. Hojfeldt, S. Bischoff, and J. Mork, "All-optical wavelength conversion and signal regeneration using an electroabsorption modulator," J. Lightwave Technol. 18, 1121-1127 (2000).

8. L. K. Oxenlowe, E. Hilliger, A. Tersigni, A. M. Nik, S. Hojfeldt, F. Romstad, K. Yvind, P. M. W. Skovgaard, K. Hoppe, and J. Hanberg, "All-optical demultiplexing and wavelength conversion in an electroabsorption modulator," in 27th European Conference on Optical Communication, 2001 (ECOC '01) (IEEE, 2001), Vol. 4, pp. 604-605.

9. K. Nishimura, M. Tsurusawa, and M. Usami, "Novel alloptical 3R regenerator using cross-absorption modulation in RF-driven electroabsorption waveguide," in 27th European Conference on Optical Communication, 2001 (ECOC '01) (IEEE, 2001), Vol. 3, pp. 286-287.

10. T. Otani, T. Miyazaki, and S. Yamamoto, "Optical 3R regenerator using wavelength converters based on electroabsorption modulator for all-optical network applications," IEEE Photon. Technol. Lett. 12, 431-433 (2000).

11. B. P. Nelson and N. J. Doran, "Optical sampling oscilloscope using nonlinear fibre loop mirror," Electron. Lett. 27, 204-205 (1991).

12. P. A. Andrekson, "Picosecond optical sampling using fourwave mixing in fibre," Electron. Lett. 27, 1440-1441 (1991).
13. S. Diez, R. Ludwig, C. Schmidt, U. Feiste, and H. G. Weber, "160-Gb/s optical sampling by gain-transparent four-wave mixing in a semiconductor optical amplifier," IEEE Photon. Technol. Lett. 11, 1402-1404 (1999).

14. S. Kawanishi, T. Yamamoto, M. Nakazawa, and M. M. Fejer, "High sensitivity waveform measurement with optical sampling using quasi-phasematched mixing in $\mathrm{LiNbO}_{3}$ waveguide," Electron. Lett. 37, 842-844 (2001).

15. P. J. Maguire, L. P. Barry, T. Krug, M. Lynch, A. L. Bradley, J. F. Donegan, and H. Folliot, "All-optical sampling utilising two-photon absorption in semiconductor microcavity," Electron. Lett. 41, 489-490 (2005).

16. P. J. Maguire, L. P. Barry, T. Krug, W. H. Guo, J. O’Dowd, M. Lynch, A. L. Bradley, J. F. Donegan, and H. Folliot, "Optical signal processing via two-photon absorption in a semiconductor microcavity for the next generation of highspeed optical communications network," J. Lightwave Technol. 24, 2683-2692 (2006).

17. M. Skold, M. Westlund, H. Sunnerud, and P. A. Andrekson, "100 GSample/s optical real-time sampling system with Nyquist-limited bandwidth," presented at the 33rd European Conference on Optical Communication, Berlin, Germany, 16-20 September 2007, post deadline paper PDP1.1.

18. M. Gicquel-Guezo, S. Loualiche, J. Even, C. Labbe, O. Dehaese, A. Le Corre, H. Folliot, and Y. Pellan, " $290 \mathrm{fs}$ switching time of Fe-doped quantum well saturable absorbers in a microcavity in $1.55 \mu \mathrm{m}$ range," Appl. Phys. Lett. 85, 5926-5928 (2004).

19. K. W. DeLong, R. Trebino, and D. J. Kane, "Comparison of ultrashort-pulse frequency-resolved-optical-gating traces for three common beam geometries," J. Opt. Soc. Am. B 11, 1595-1608 (1994).

20. P.-A. Lacourt, M. Hanna, and J. M. Dudley, "Broad-band and ultrasensitive pulse characterization using frequencyresolved optical gating via four-wave mixing in a semiconductor optical amplifier," IEEE Photon. Technol. Lett. 17, 157-159 (2005).

21. M. D. Thomson, J. M. Dudley, L. P. Barry, and J. D. Harvey, "Complete pulse characterization at $1.5 \mu \mathrm{m}$ by cross-phase modulation in optical fibers," Opt. Lett. 23, 1582-1584 (1998).

22. C. Dorrer and I. Kang, "High-sensitivity spectrographic method for characterizing picosecond optical pulses and data modulators," in Optical Fiber Communication Conference and Exhibit, 2002 (OFC 2002) (IEEE, 2002), pp. FD4-1-FD4-3.

23. D. J. Kane, "Real-time measurement of ultrashort laser pulses using principal component generalized projections," IEEE J. Sel. Top. Quantum Electron. 4, 278-284 (1998).

24. D. Reid and J. Harvey, "Linear spectrograms using electrooptic modulators," IEEE Photon. Technol. Lett. 19, 535-537 (2007). 